\title{
Anti-beta 2 glycoprotein 1 domain IgA positive antiphospholipid syndrome secondary to incomplete Behcet's disease: a case report
}

\author{
Lili Dai ${ }^{1 \#}$, Jing Chen ${ }^{2 \#}$, Zhengli Chen ${ }^{1}$, Shumin Zhu ${ }^{1}$, Yingqi Zhang ${ }^{3}$, Yulan Geng ${ }^{1}$, Zhanqing Gu ${ }^{4}$ \\ ${ }^{1}$ Department of Laboratory Medicine, the First Hospital of Hebei Medical University, Shijiazhuang, China; ${ }^{2}$ Department of Blood Transfusion, \\ the Third Hospital of Hebei Medical University, Shijiazhuang, China; ${ }^{3}$ Department of Emergency, the First Hospital of Hebei Medical University, \\ Shijiazhuang, China; ${ }^{4}$ Department of Rheumatology, the First Hospital of Hebei Medical University, Shijiazhuang, China \\ \#These authors contributed equally to this work. \\ Correspondence to: Yulan Geng, MD. Department of Laboratory Medicine, the First Hospital of Hebei Medical University, 89 Donggang Road, \\ Shijiazhuang 050031, China. Email: ylgeng123@163.com; Zhanqing Gu, MD. Department of Rheumatology, the First Hospital of Hebei Medical \\ University, 89 Donggang Road, Shijiazhuang 050031, China. Email: gzhq1964@163.com.
}

\begin{abstract}
Antiphospholipid syndrome (APS) is an autoimmune systemic disorder characterized by arterial, venous, or small vessel thrombosis, and/or recurrent early pregnancy loss, fetal loss, or pregnancy morbidity. APS is induced by persistent positive antiphospholipid antibodies (aPL), the main being lupus anticoagulant (LA), and/or anticardiolipin (aCL) antibodies, and/or anti-beta 2 glycoprotein 1 ( $\beta 2$-GP1) antibodies. Some studies have shown that the incidence of APS is about 5 new cases per 100,000 persons per year, and the prevalence is around 40-50 cases per 100,000 persons. APS can be primary or secondary. Secondary APS often coexists with another autoimmune disorder, most commonly systemic lupus erythematosus (SLE). Behcet's disease (BD) is usually characterized by recurrent oral and genital aphthous ulcers and ocular involvement. It can occasionally affect the venous system. BD usually affects small vessels, but can sometimes affect large veins or even a variety of veins. Because most of APS is secondary to SLE, APS secondary to incomplete $\mathrm{BD}$ is quite rare. This report describes a case in which a 15 -year-old male experienced bilateral leg swelling and pain. The patient had a long history of self-healing recurrent mouth ulcers. Laboratory tests revealed positive $\beta 2-\mathrm{GP} 1$ immunoglobulin A (IgA). His symptoms improved by using steroids, prednisolone, uro-kinase, and hirudin. In this rare case of secondary APS, the patient was diagnosed with anti- $\beta 2-G P-1$ IgA positive to incomplete BD. It is a rare case of secondary APS with positive anti- $\beta 2-G P 1$ IgA to incomplete BD. It is suggested that patients with recurrent mouth ulcers should be closely examined to prevent thrombosis, and more laboratory markers should be used to avoid a risk of misdiagnosing patients with APS.
\end{abstract}

Keywords: Antiphospholipid (antibody) syndrome (APS); Behcet's disease (BD); thrombosis; anti-beta 2 glycoprotein 1 domain IgA ( $\beta 2-G P 1 \operatorname{IgA})$; case report

Submitted Mar 12, 2021. Accepted for publication May 19, 2021.

doi: $10.21037 /$ apm-21-711

View this article at: http://dx.doi.org/10.21037/apm-21-711

\section{Introduction}

Antiphospholipid (aPL) antibody syndrome, or antiphospholipid syndrome (APS), is an autoimmune systemic disorder characterized by arterial and venous thrombosis, pathological pregnancy (early abortion and stillbirth in middle and late pregnancy), thrombocytopenia and other symptoms with positive laboratory indicators of aPL antibodies, including lupus anticoagulant (LA), immunoglobulin $G(\operatorname{IgG})$, immunoglobulin $M(\operatorname{IgM})$, and immunoglobulin A (IgA) anticardiolipin (aCL) antibodies, and anti-beta 2 glycoprotein 1 domain ( $\beta 2-G P 1)(1-3)$. The clinical manifestations of APS include livedo reticularis, 

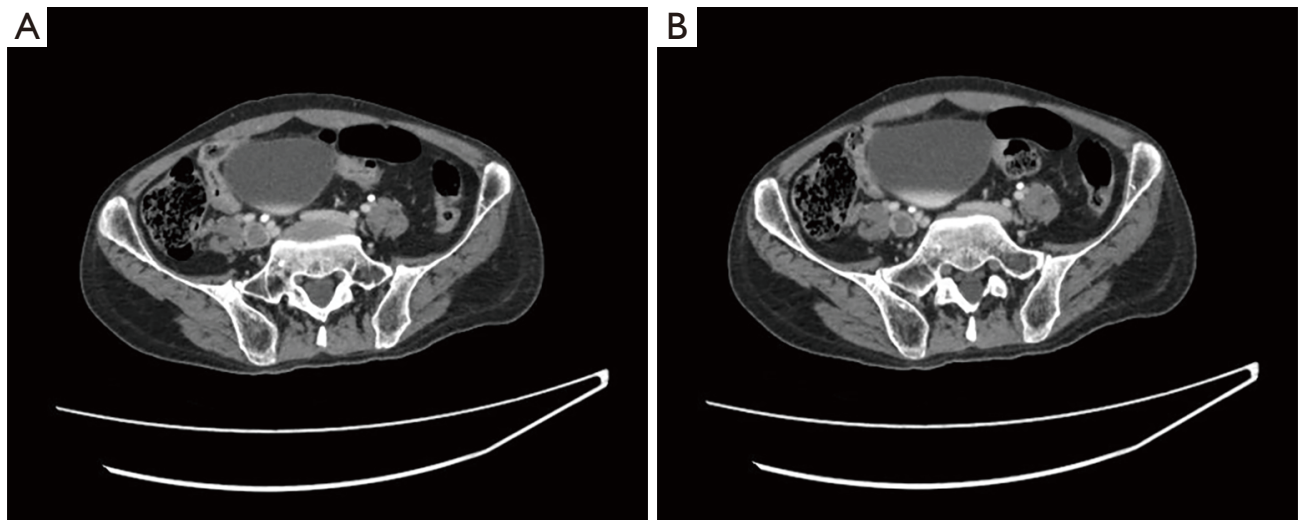

Figure 1 Two different levels by CT scanning for larger diameter and turgor defect of right common iliac vein compared with left common iliac vein.

cutaneous ulcerations, thrombocytopenia, hemolytic anemia, valvular heart disease, and nephropathy. According to the most recent classification criteria in 2006, APS is identified by persistent antiphospholipid antibodies including LA, aCL and $\beta 2$-GP1 >12 weeks with imaging or pathologic support for arterial or venous thrombosis or having a history of pathological pregnancy (4). Behcet's disease (BD) is a multifactorial systemic inflammatory autoimmune disease with recurrent oral, genital, and anal ulcers, and dermatologic manifestations, such as erythema nodosum. Vascular involvement is one of the relatively rare complications of $\mathrm{BD}$, and usually suggests a poor prognosis. BD may involve a variety of vessels, and commonly affects small veins, but can sometimes affect large veins (5). Among autoimmune diseases, systemic lupus erythematosus (SLE) was found to be the main cause of thrombotic disorder; however, it has been reported that aPLs are also present in patients with BD (6). This report describes a case of a 15 -year-old APS patient with anti- $\beta 2-$ GP 1 IgA positive associated with incomplete BD who developed bilateral lower extremities swelling and pain from the thrombotic occlusion of several veins. We present the following article in accordance with the CARE reporting checklist (available at http://dx.doi.org/10.21037/apm-21-711).

\section{Case presentation}

A 15 -year-old male was hospitalized in the Rheumatology Department for swelling and pain of both lower extremities. The chief complaint revealed that the patient had been to three different hospitals due to recurrent swelling and pain of the lower extremities over the past 5 months.
To eliminate the swelling, the patient had undergone a number of treatments, including topical plasters of Chinese medicine, balloon dilatation, and catheter-directed thrombolysis, while the symptoms were not improved significantly. The patient also reported a history of selfhealing recurrent mouth ulcers accompanied by a burning pain that he had experienced since he was 8 years old. His father and brother also had a history of self-healing recurrent mouth ulcers.

The physical examination showed the patient had a tachycardia of 96 beats per minute, a blood pressure of $110 / 89 \mathrm{mmHg}$, a respiration rate of 18 breaths per minute, and a temperature of $37^{\circ} \mathrm{C}$. Skin examination showed that the color of his skin and mucosa were normal; however, the patient presented with 3 painful mouth ulcers with pale yellow pseudomembrane, and a $2-4 \mathrm{~cm}$ red pot with subcutaneous nodules on the fibular side of his left lower limb. His right lower limb, which was swollen and painful, was $46 \mathrm{~cm}$ in circumference, and longer than his left limb, which was $44 \mathrm{~cm}$. Cardiac auscultation revealed a regular rhythm, and no murmurs were found. The auscultating lungs sounds were clear. The abdominal and neurological examinations were normal.

An initial computed tomography (CT) abdominal and pelvic scan showed (see Figure 1): (I) an increased diameter and turgor defect of the bilateral common iliac vein; (II) Coarse and thickening walls of the inferior vena cava and, a iliac vein surrounded by unclear fat; (III) extensive retroperitoneal tortuous vascular shadows; (IV) blurred retroperitoneal fat clearance; (V) swelling of the right psoas muscle; (VI) little effusion in the abdominal and pelvic cavity; and (VII) multiple small retroperitoneal lymph 

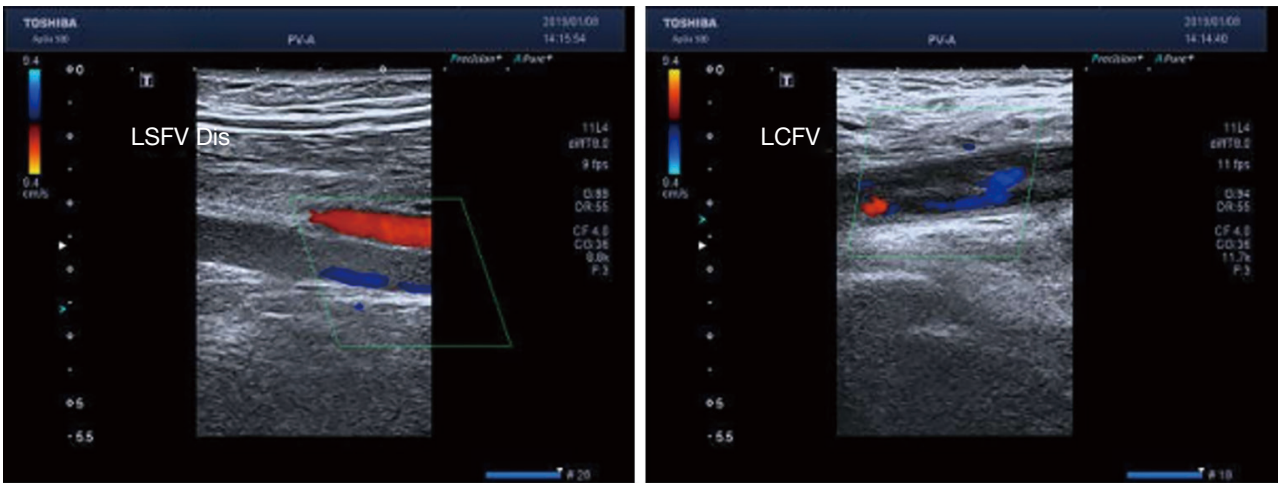

Figure 2 Ultrasonography of bilateral limbs, revealing thrombosis of the left common femoral vein (LCFV), the left superficial femoral vein (LSFV), and the popliteal vein. Dis, distal LSFV from the heart.

nodes.

The ultrasound examination of the bilateral limbs showed: (I) thrombosis of the left common femoral vein (LCFV), the left superficial femoral vein (LSFV), and the popliteal vein (see Figure 2); (II) hypercoagulability in the left tibiofibular trunk vein, the left posterior veins, and the right lower extremity veins; and (III) thrombosis of the distal vessels of the inferior vena cava, the right common iliac vein, the left common iliac vein, and a proximal segment of the left internal iliac vein, and left external iliac vein.

Laboratory examinations revealed the following: white blood cell count: $7.2 \times 10^{9} / \mathrm{L}$; hemoglobin: $101 \mathrm{~g} / \mathrm{L}$; mean corpuscular volume: $77.4 \mathrm{fL}$; mean corpuscular hemoglobin: 23.0 pg; mean corpuscular hemoglobin concentration: $297 \mathrm{~g} / \mathrm{L}$; platelet count: $306 \times 10^{9} / \mathrm{L}$; erythrocyte sedimentation rate (ESR): $94 \mathrm{~mm} / \mathrm{h}$; C-reactive protein (CRP): $40.5 \mathrm{mg} / \mathrm{L}$; prealbumin: $72 \mathrm{mg} / \mathrm{L}$; folic acid: $2.8 \mathrm{ng} / \mathrm{mL}$; direct antiglobulin test (Coombs test): negative; Ham test and Sugarwater test: negative; LA: 1.11; Protein C (PC) activity: 60\%; Protein S (PS) activity: 48\%; prothrombin time (PT): $23.1 \mathrm{~s}$; PT activity: $35 \%$; international normalized ratio (INR): 2.07; D-Dimer: $1,000 \mathrm{mg} / \mathrm{L}$; all aCLs isotypes: negative; IgA anti- $\beta 2-G P 1: 40 \mathrm{RU} / \mathrm{mL}$, total IgG and IgM anti- $\beta 2-$ GP1: negative; antinuclear antibody IgG: weak positive (tilter: 1:100) and granular isotype; serine protease 3 antibody, myeloperoxidase antibody: negative; blood culture with and without oxygen: negative; T. SPOT-TB: negative; serum IgA: $4.10 \mathrm{~g} / \mathrm{L}$.

Based on the presence of self-healing recurrent mouth ulcers only, family history of mouth ulcers, and young age, hypercoagulable state which showed with recurrent thrombosis in the past year, positive IgA anti- $\beta 2-G P 1$, and low activity of PC and PS, it was highly considered as APS associated with incomplete BD. The patient had been receiving oral anticoagulation therapy with warfarin to target INR 2.0-3.0 since he was diagnosed thrombosis in the first hospital. In our hospital, folacin, calcium carbonate, dexamethasone, prednisolone, cyclophosphamide (because of moderate risk of azathioprine), hirudin, uro-kinase were also prescribed for anemia, lower serum calcium level, immunity inhibition, anti-inflammation, antiplatelet aggregation, anti-coagulation, and thrombolysis, respectively.

One month later, his general symptoms were markedly improved. He was discharged home with a regular steroid, cyclophosphamide and warfarin to target INR 2.0-3.0. Three months later, his condition kept better. And the serum IgA anti- $\beta 2-G P 1$ was $46 \mathrm{RU} / \mathrm{mL}$ while activity of PC and PS was $63 \%$ and $45 \%$, respectively, which were with no obvious improvement. However, the patient had no symptoms or signs of SLE longer. Based on his history, symptoms, age, positive IgA anti- $\beta 2-\mathrm{GP} 1$ of 12 weeks interval, and low activity of PC and PS, he was finally diagnosed as APS secondary to incomplete BD.

All procedures performed in studies involving human participants were in accordance with the ethical standards of the institutional and/or national research committee(s) and with the Helsinki Declaration (as revised in 2013). Written informed consent was obtained from the patient.

\section{Discussion}

APS is an autoimmune disorder characterized by the presence of clinical features and specific circulating aPL autoantibodies. The modified Sapporo criteria require that 
at least one clinical and one laboratory criterion emerge, and laboratory evidence requires a confirmatory test 12 weeks after an initial positive test (7). Some estimates indicate that the incidence of APS is around 5 new cases per 100,000 persons per year, and the prevalence is around $40-50$ cases per 100,000 persons (8). In fact, among the 100,000 people aged $\geq 18$ years, the annual incidence and prevalence of APS has been reported to be about 2.1 and 50, respectively, and no difference has been found between the sexes (9). Deep venous thrombosis has been reported to be the most frequent clinical manifestation of APS in patients (9). It has been reported in the literature that APS can be classified as two clinical isotypes: primary or secondary APS. Many diseases may cause secondary APS, such as SLE (9-11), rheumatoid arthritis (12), or Sjögren syndrome (13). BD is also a cause of secondary APS (14). Treatment for APS usually conclude primary thromboprophylaxis, secondary thromboprophylaxis, catastrophic APS therapy and treatment of obstetric APS. Low-dose aspirin (LDA) and hydroxychloroquine (HCQ) is often used in the primary thromboprophylaxis, and high-intensity warfarin combined with antiplatelet therapy or the statin therapy is often used in the secondary thromboprophylaxis. For catastrophic APS therapy, the treatment of any underlying condition, such as infection, and "triple therapy" are essential, which include intravenous heparin, high dose steroid (for systemic inflammatory response syndrome), and intravenous immunoglobulin (IVIG), and/or plasmapheresis. While for the treatment of obstetric APS, every aPL-positive patient should be treated with LDA to prevent preeclampsia and treated with combination LDA and prophylactic heparin therapy to prevent recurrent pregnancy loss (15).

aPLs are a group of heterogeneous antibodies with specificity for binding to negatively charged phospholipids on endothelial cell surfaces. Among them, aCLs are associated with thrombosis in secondary APS in BD patients (5). In the present case, a BD patient presented with a long-term and family history of mouth ulcers, oral mucosa being the only ulcer area, so called incomplete BD. It has been suggested that isolated IgA anti- $\beta 2-\mathrm{GP} 1$ may have accelerated his thrombogenesis. According to some research, BD is thought to be related to severe thrombotic disorder because, similar to SLE, it is characterized by an increased risk of arterial and venous thrombosis (16-19). SLE patients with isolated IgA anti- $\beta 2-G P 1$ have been reported to experience significantly more thromboembolic events, higher titers of IgA anti- $\beta 2-G P 1$ have been found in the serum of patients who have experienced a thromboembolic event, and isolated IgA anti- $\beta 2-\mathrm{GP} 1$ has been found to be markedly associated with an increased prevalence of diseases (16). The anticoagulation system also plays a critical role in preventing thrombosis. Patients with significantly low PC and PS activity are unable to effectively inhibit blood coagulation, which can easily result in thromboembolism. As the patient had normal liver and kidney function, the question of whether PC and PS antibodies, which are included in autoimmune aPL antibodies, cause a reduction in activity needs to be investigated further.

BD patients with isolated anti- $\beta 2-G P 1$ IgA positivity may experience thromboembolisms for a number of reasons. First, BD may increase the risk of thrombosis that does not depend on aPLs $(20,21)$. Second, during the active stage of various autoimmune diseases, the coexisting inflammation reaction and the activation of innate immunity may act as a necessary "second hit" that induces thrombosis (22). Notably, in the present case, the patient had elevated ESR and CRP, and decreased prealbumin, which shows the role of inflammation in thrombosis. Third, IgA anti- $\beta 2-G P 1$ may have different phenotypes, which used to depend upon the concentration of circling aPLs in systemic autoimmunity. For example, among anti- $\beta 2$ GP1, antibodies that recognize domain I of $\beta 2-G P 1$ are predictive of thrombosis, while those that recognize other domains are not (23). Fourth, the neutrophil release of chromatin in the form of neutrophil extracellular traps is an important contributor to thrombosis $(24,25)$. Finally, anticoagulant and fibrinolytic disorders in BD patients result in diminished fibrinolysis (26).

Research has shown that insufficiency or a lack of natural anticoagulant proteins, including PC, PS, and antithrombin, play a role in thrombosis among BD patients $(27,28)$. In the present case, the prolonged PT may be related to the warfarin treatment, the elevated D-dimer may be linked to using uro-kinase, and the low activity of PC and PS may have been the cause of the thrombosis event. The mechanisms by which aPLs are associated with thrombosis include abnormal platelet aggregation, decreased endothelial cell prostacyclin production, increased adhesive neutrophils mediated by beta- 2 integrin Mac-1, the inhibition of PC, and decreased fibrinolysis $(29,30)$. The evidence linking aPLs to the thrombotic events in patients with SLE is compelling (31); however, a meta-analysis established that compared to controls, patients with BD have a significantly higher prevalence of aPLs (i.e., aCL and anti- $\beta 2-$-GPI antibodies) (32). Some studies have suggested 
that aPLs may be associated with the vascular thrombosis observed in BD patients $(33,34)$. Notably, Hull et al. found that 13 of $70 \mathrm{BD}$ patients had positive aCL, and suggested a correlation between aCL and thrombotic events (35). Similar to SLE, these results suggest that APS may be involved in BD. In relation to thrombosis, this mechanism may also have affected the present case and other cases of patients with $\mathrm{BD}$, such that an occlusion or thrombosis are accompanied by elevated aCL, LA, and anti- $\beta 2-G P 1(28,36)$. There is no direct evidence that steroid therapy can prevent the recurrence of thrombosis in APS patients; however, it may suppress the aPL titers. In the present case, steroid therapy was primarily chosen to alleviate the symptoms of BD. At the same time, cyclophosphamide was used to suppress autoimmunity and warfarin for anti-thrombosis.

In summary, patients with recurrent mouth ulcers may have BD. Anti- $\beta 2-\mathrm{GP} 1 \mathrm{IgA}$ positive APS secondary to incomplete $\mathrm{BD}$ is rare, which may play an important role in the pathogenesis of severe thrombotic events. Additionally, we suggest that patients with mouth ulcers are given more attention to prevent thrombosis, and that more laboratory markers are used to avoid the risk of a misdiagnosis of secondary APS.

\section{Patient perspective}

I had been disturbed by recurrent swelling and pain of the lower extremities for 5 months without any reasons. I had undergone a number of treatments, including topical plasters of Chinese medicine, balloon dilatation, and catheter-directed thrombolysis, while the symptoms were not improved significantly. Finally, doctors made a definite diagnosis of anti- $\beta 2-G P 1$ IgA positive APS secondary to incomplete $\mathrm{BD}$, contributing to effective treatment with cyclophosphamide and warfarin. This kind of treatment method improved my symptoms.

\section{Acknowledgments}

Funding: None.

\section{Footnote}

Reporting Checklist: The authors have completed the CARE reporting checklist. Available at http://dx.doi.org/10.21037/ apm-21-711

Conflicts of Interest: All authors have completed the ICMJE uniform disclosure form (available at http://dx.doi. org/10.21037/apm-21-711). The authors have no conflicts of interest to declare.

Ethical Statement: The authors are accountable for all aspects of the work in ensuring that questions related to the accuracy or integrity of any part of the work are appropriately investigated and resolved. All procedures performed in studies involving human participants were in accordance with the ethical standards of the institutional and/or national research committee(s) and with the Helsinki Declaration (as revised in 2013). Written informed consent was obtained from the patient.

Open Access Statement: This is an Open Access article distributed in accordance with the Creative Commons Attribution-NonCommercial-NoDerivs 4.0 International License (CC BY-NC-ND 4.0), which permits the noncommercial replication and distribution of the article with the strict proviso that no changes or edits are made and the original work is properly cited (including links to both the formal publication through the relevant DOI and the license). See: https://creativecommons.org/licenses/by-nc-nd/4.0/.

\section{References}

1. Whitaker KL. Antiphospholipid antibody syndrome: The difficulties of diagnosis. JAAPA 2017;30:10-4.

2. Bertolaccini ML, Amengual O, Andreoli L, et al. 14th International Congress on Antiphospholipid Antibodies Task Force. Report on antiphospholipid syndrome laboratory diagnostics and trends. Autoimmun Rev 2014;13:917-30.

3. Ruiz-García R, Serrano M, Martínez-Flores JÁ, et al. Isolated IgA anti-b2 glycoprotein I antibodies in patients with clinical criteria for antiphospholipid syndrome. J Immunol Res 2014;2014:704395.

4. Mezhov V, Segan JD, Tran H, et al. Antiphospholipid syndrome: a clinical review. Med J Aust 2019;211:184-8.

5. Mendoza-Pinto C, García-Carrasco M, JiménezHernández M, et al. Etiopathogenesis of Behcet's disease. Autoimmun Rev 2010;9:241-5.

6. Kandolf-Sekulovic L, Pavlovic MD, Glisic B, et al. Adamantiades-Behçet's disease, deep venous thrombosis and anticardiolipin antibodies: report of two cases. J Eur Acad Dermatol Venereol 2005;19:484-6.

7. The Sydney classification criteria for definite antiphospholipid syndrome rheumatology network, 
Available online: http://www.rheumatologynet-work. com/fibromyalgia/sydney-classification-criteria-definiteantiphospholipid-syndrome (accessed 3 February 2018).

8. Cervera R. Antiphospholipid syndrome. Thromb Res 2017;151 Suppl 1:S43-S47.

9. Duarte-García A, Pham MM, Crowson CS, et al. The epidemiology of antiphospholipid sysdrome. A populationbased study. Arthritis Rheumatol 2019;71:1545-52.

10. Pons-Estel GJ, Andreoli L, Scanzi F, et al. The antiphospholipid syndrome in patients with systemic lupus erythematosus. J Autoimmun 2017;76:10-20.

11. El-Moniem GA, El-Garf K, Sobhy N, et al. Characterization of the Clinical and Laboratory Features of Primary and Secondary Antiphospholipid Syndrome in a Cohort of Egyptian Patients. Curr Rheumatol Rev 2020;16:304-10.

12. Chung WS, Peng CL, Lin CL, et al. Rheumatoid arthritis increases the risk of deep vein thrombosis and pulmonary thromboembolism: a nationwide cohort study. Ann Rheum Dis 2014;73:1774-80.

13. Chung WS, Lin CL, Sung FC, et al. Increased risks of deep vein thrombosis and pulmonary embolism in Sjogren syndrome: a nationwide cohort study. J Rheumatol 2014;41:909-15.

14. Calamia KT, Schirmer M, Melikoglu M. Major vessel involvement in Behcet's disease: an update. Curr Opin Rheumatol 2011;23:24-31.

15. Sammaritano LR. Antiphospholipid syndrome. Best Pract Res Clin Rheumatol 2020;34:101463.

16. Sweiss NJ, Bo R, Kapadia R, et al. IgA anti- $\beta 2$ Glycoprotein I autoantibodies are associated with an increased risk of thromboembolic events in patients with systemic lupus erythematosus. PLoS One 2010;5:e12280.

17. Hughes GRV. The antiphospholipid syndrome: Ten years on. Lancet 1993;342:341-4.

18. Meijide H, Sciascia S, Sanna G, et al. The clinical relevance of IgA anticardiolipin and IgA anti- $\beta 2$ glycoprotein I antiphospholipid antibodies: a systematic review. Autoimmun Rev 2013;12:421-5.

19. Danowski A, Kickler TS, Petri M. Anti-beta2-glycoprotein I: prevalence, clinical correlations, and importance of persistent positivity in patients with antiphospholipid syndrome and systemic lupus erythematosus. J Rheumatol 2006;33:1775-9.

20. Frodlund M, Vikerfors A, Grosso G, et al. Immunoglobulin A anti-phospholipid antibodies in Swedish cases of systemic lupus erythematosus: associations with disease phenotypes, vascular events and damage accrual. Clin Exp Immunol 2018;194:27-38.

21. Romero-Díaz J, Garcia-Sosa I, Sanchez-Guerrero J. Thrombosis in systemic lupus erythematosus and other autoimmune diseases of recent onset. J Rheumatol 2009;36:68-75.

22. Asanuma Y, Oeser A, Shintani AK, et al. Premature coronary-artery atherosclerosis in systemic lupus erythematosus. New Eng J Med 2003;349:2407-15.

23. Levine JS, Branch DW, Rauch J. Anti-phospholipid syndrome. N Engl J Med 2002;346:752-63.

24. Radic M, Pattanaik D. Cellular and Molecular Mechanisms of Anti-Phospholipid Syndrome. Front Immunol 2018;9:969.

25. Zuo Y, Yalavarthi S, Gockman K, et al. Anti-Neutrophil Extracellular Trap Antibodies and Impaired Neutrophil Extracellular Trap Degradation in Antiphospholipid Syndrome. Arthritis Rheumatol 2020;72:2130-5.

26. Shang H, Ye JJ, Ji M, et al. Anticoagulant and Fibrinolytic Disorders in Patients with Behçet's Disease and Recurrent Aphthous Ulcer. Chin J Physiol 2011;54:235-40.

27. de Laat B, Derksen RH, Urbanus RT, et al. IgG antibodies that recognize epitope Gly40-Arg43 in domain I of beta 2-glycoprotein I cause LAC, and their presence correlates strongly with thrombosis. Blood 2005;105:1540-5.

28. Jeong H, Yoo IK, Choi S, et al. Thrombosis in Behçet's disease: a Behçet's disease patient with complete thrombotic obstruction of IVC and both iliac veins and decreased protein $S$ activity. Rheumatol Int 2013;33:1633-5.

29. Lenk N, Ozet G, Alli N, et al. Protein C and protein S activities in Behcet's disease as risk factors of thrombosis. Int J Dermatol 1998;37:124-5.

30. Sule G, Kelley WJ, Gockman K, et al. Increased adhesive potential of antiphospholipid sysdrome neutrophils mediated by beta- 2 integrin Mac-1. Arthritis Rheumatol 2020;72:114-24.

31. Saadoun D, Wechsler B. Behcet's disease. Orphanet J Rare Dis $2012 ; 7: 20$.

32. Islam MA, Alam SS, Kundu S, et al. Prevalence of antiphospholipid antibodies in Behçet's disease: A systematic review and meta-analysis. PLoS One 2020;15:e227836.

33. Balta S, Balta I, Ozturk C, et al. Behçet's disease and risk of vascular events. Curr Opin Cardiol 2016;31:451-7.

34. Hatemi G, Christensen R, Bang D, et al. 2018 update of the EULAR recommendations for the management of Behçet's syndrome. Ann Rheum Dis 2018;77:808-18.

35. Hull RG, Harris EN, Charavi AE, et al: Anticardiolipin 
antibodies: Occurrence in Behçet's syndrome. Ann Rheum Dis $1984 ; 43: 746-8$.

36. Mukai Y, Tsutsui H, Todaka K, et al. Total occlusion of inferior vena cava in a patient with antiphospholipid

Cite this article as: Dai L, Chen J, Chen Z, Zhu S, Zhang Y, Geng Y, Gu Z. Anti-beta 2 glycoprotein 1 domain IgA positive antiphospholipid syndrome secondary to incomplete Behcet's disease: a case report. Ann Palliat Med 2021;10(6):7114-7120. doi: 10.21037/apm-21-711 antibody syndrome associated with Behçet's disease. Jpn Circ J 2001;65:837-8.

(English Language Editor: L. Huleatt) 